\title{
Systematic Study of Au-Au Collisions with AGS Experiment E917
}

\author{
B. Holzman ${ }^{\mathrm{a}}$, for the E917 Collaboration
}

B.B. Back ${ }^{\mathrm{b}}$, R.R. Betts ${ }^{\mathrm{a}}$, J. Chang ${ }^{\mathrm{c}}$, W.C. Chang ${ }^{\mathrm{c}}$, C.Y. Chi ${ }^{\mathrm{d}}$, Y.Y. Chu ${ }^{\mathrm{e}}$, J.B. Cumming ${ }^{\mathrm{e}}$, J.C. Dunlop ${ }^{f}$, W. Eldredge ${ }^{c}$, S.Y. Fung ${ }^{c}$, R. Ganz ${ }^{a}$, E. Garcia ${ }^{g}$, A. Gillitzer ${ }^{b}$, G. Heintzelman ${ }^{f}$,

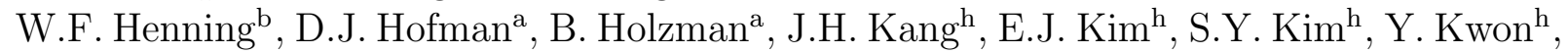
D. McLeod ${ }^{\mathrm{a}}$, A.C. Mignerey ${ }^{\mathrm{g}}$, M. Moulson ${ }^{\mathrm{d}}$, V. Nanal ${ }^{\mathrm{b}}$, C.A. Ogilvie ${ }^{\mathrm{f}}$, R. Pak ${ }^{\mathrm{i}}$, A. Ruangma ${ }^{\mathrm{g}}$, D. Russ ${ }^{g}$, R.K. Seto ${ }^{c}$, P.J. Stanskas ${ }^{g}$, G.S.F. Stephans ${ }^{f}$, H.Q. Wang ${ }^{\mathrm{c}}$, F.L.H. Wolfs ${ }^{\mathrm{i}}$, A.H. Wuosmaa ${ }^{\mathrm{b}}$, H. Xiang ${ }^{\mathrm{c}}$, G.H. Xuc ${ }^{\mathrm{c}}$, H.B. Yao ${ }^{\mathrm{f}}$, C.M. Zou ${ }^{\mathrm{c}}$

${ }^{a}$ University of Illinois at Chicago, Chicago, IL 60607

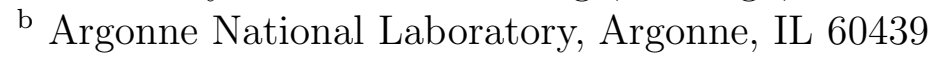

${ }^{\text {c }}$ University of California, Riverside, CA 92521

${ }^{d}$ Nevis Laboratories, Columbia University, Irvington, NY 10533

e Brookhaven National Laboratory, Upton, NY 11973

${ }^{\mathrm{f}}$ Massachusetts Institute of Technology, Cambridge, MA 02139

${ }^{g}$ University of Maryland, College Park, MD 20742

${ }^{\text {h }}$ Yonsei University, Seoul 120-749, South Korea

${ }^{\text {i }}$ University of Rochester, Rochester, NY 14627

The systematics of baryon stopping and strange particle production have been studied in $\mathrm{Au}+\mathrm{Au}$ collisions at 6,8 , and $10.8 \mathrm{GeV}$ per nucleon with AGS experiment E917. In particular, the systematic behavior of the proton yields, $\phi$ meson production, and the yields of antilambdas and antiprotons are examined as functions of beam energy and centrality.

\section{INTRODUCTION}

In just the last year, center-of-mass energies reached in heavy ion collisions have increased by more than an order of magnitude over those previously attained at the AGS. Nevertheless, several outstanding issues remain in the AGS energy regime. The characterization of the evolution of the initial-state nucleons can be studied through the spectra and rapidity distribution of protons. The mechanisms underlying the production of strange and anti-strange quarks may be investigated through the examination of the yields and spectral shapes of $\phi$ mesons, antilambdas, and antiprotons.

${ }^{*}$ This work was supported by the U. S. Department of Energy, the National Science Foundation (USA) and KOSEF (Korea). 


\section{EXPERIMENT}

E917 is the last in the E802/E859/E866 series of heavy ion experiments that were performed at the AGS. The E917 apparatus consisted of a series of beam counters, a beam vertexing detector, a multiplicity array surrounding the target, a hodoscope, a zero-degree calorimeter, and a movable spectrometer composed of drift chambers, magnet, and timeof-flight wall. The calorimeter and multiplicity array were used to determine collision centrality, while the spectrometer was used for tracking and particle identification. Detailed descriptions of the equipment can be found in [1, 2].

\section{PROTON YIELDS}

The invariant yields of protons were determined from 6, 8, and $10.8 \mathrm{GeV} /$ nucleon $\mathrm{Au}+\mathrm{Au}$ collisions as a function of transverse mass $\left(m_{T}=\sqrt{m_{0}^{2}+p_{T}^{2}}\right)$. The data were fit with a Boltzmann function over ten rapidity intervals. Rapidity distributions $(d N / d y)$ were extracted for five centrality classes at each beam energy and are presented in Figure 1 .

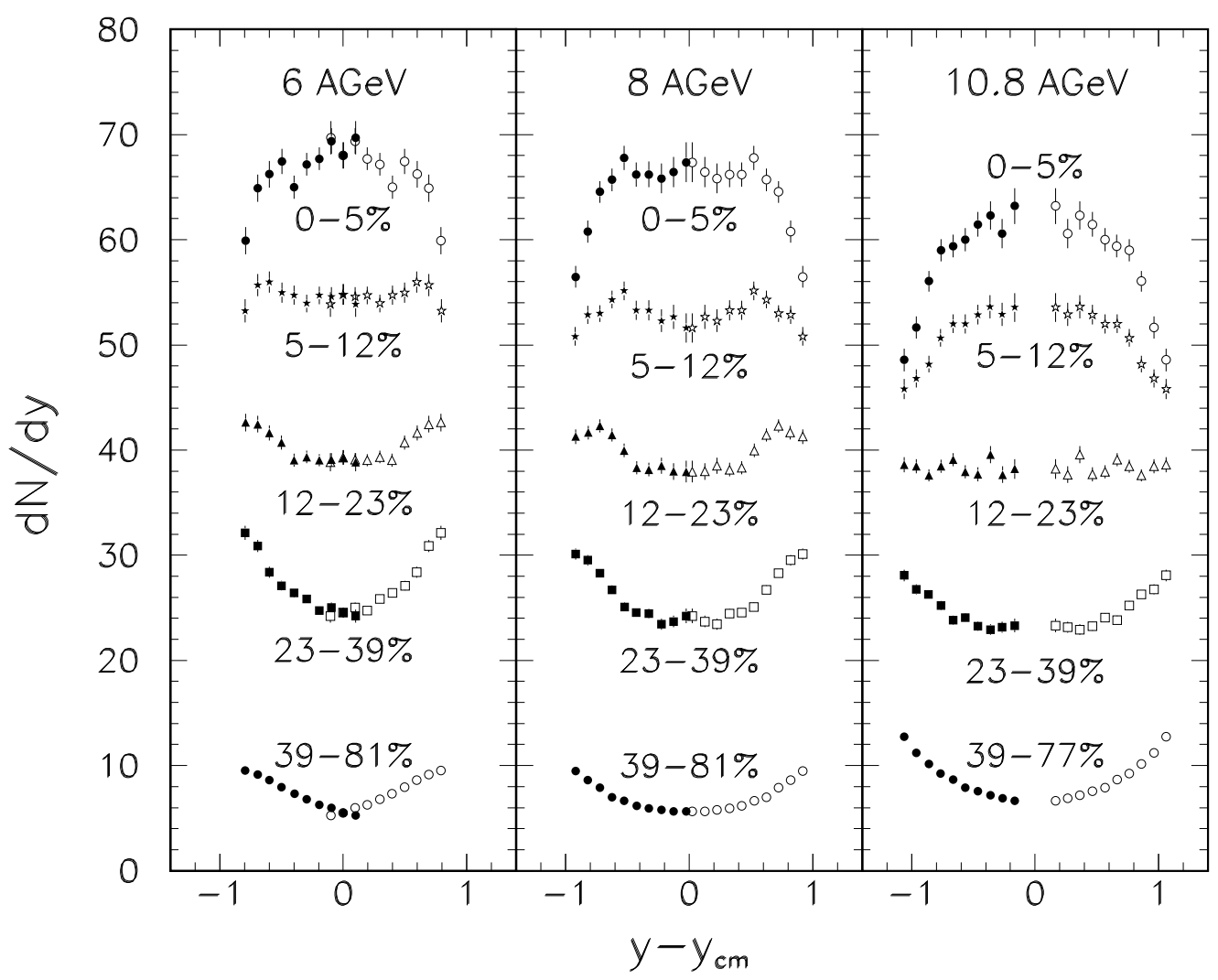

Figure 1. Proton rapidity distributions at three beam energies for five centrality classes. Open points are the measured data reflected about mid-rapidity. 
Two qualitative features emerge from the data. First, a smooth evolution in shape from concave for the most peripheral event class $(39 \%-77 / 81 \%)$ to convex for the most central $(0-5 \%)$ is evident at all beam energies. Second, the $d N / d y$ curves are not well-described by a singly-peaked function, but rather are consistent with a bimodal distribution, even for the most central events. The nature of this double-peak has been quantified by fitting the curves with a Gaussian distribution reflected about mid-rapidity [3]. The persistence of bimodal shape for even the most central events suggests that complete stopping is not achieved at AGS energies, and that the longitudinal rapidity distribution is a result of transparency.

\section{ANTILAMBDA/ANTIPROTON RATIO}

A measurement of the ratio of antilambdas $(\bar{\Lambda})$ to directly produced antiprotons $\left(\bar{p}_{\text {direct }}\right)$ allows us to indirectly compare the ratio of $\bar{s}$ quarks to light anti-quarks $(\bar{u}, \bar{d})$. Theoretical predictions for this quantity range from 0.9 from thermal models [4] to 1.3 in the RQMD cascade model [5].

Invariant mass spectra for accepted $\bar{p} \pi^{+}$pairs were constructed for bins in $m_{T}$ and $y$ for both central $(0-12 \%)$ and peripheral $(12-77 \%)$ collisions at a beam energy of 10.8 $\mathrm{GeV} /$ nucleon for events which contained at least one $\bar{p}$ in the spectrometer. An eventmixed background was normalized to the mass region outside the $\bar{\Lambda}$ peak, defined as lying more than 6 standard deviations away from the nominal $\bar{\Lambda}$ mass. The yield was determined by subtracting the number of $\bar{p} \pi^{+}$pairs which lie within 3 standard deviations of the peak from those in the background. Since a significant number of the observed antiprotons are produced by $\bar{\Lambda}$ decay, both $m_{T}$ spectra were simultaneously fit with Boltzmann functions, as described in [6]. This fit procedure yields values for $d N / d y_{\bar{\Lambda}}, \bar{\Lambda} / \bar{p}_{\text {direct }}$, and the inverse slopes for each.

We find that the $\bar{\Lambda} / \bar{p}$ ratio increases from $0.26_{-.15-.4}^{+.19+.5}$ in peripheral events to $3.6_{-1.8-1.1}^{+4.7+2.7}$ in central events. This is consistent with an earlier indirect estimate which combined different results for the $\bar{p}$ yield from the E864 and E878 experiments, under the assumption that the difference was due to different acceptances for $\bar{p}$ from $\bar{\Lambda}$ feed-down [7].

\section{PHI MESON YIELDS}

The $\phi$ meson is the lightest bound state of two strange quarks $(s \bar{s})$; it is therefore a useful probe of the strangeness produced in the reaction. It's properties may also be sensitive to in-medium modifications of meson properties.

$\phi$ mesons were reconstructed from $K^{+} K^{-}$pairs in the data in a similar fashion to that of the $\bar{\Lambda}$. The $\phi m_{T}$ spectra were fit with exponential functions, and the yields were extracted over the rapidity range $1.2 \leq y \leq 1.6$ for five different centrality classes. The absolute yields as a function of centrality have been previously reported [8]. Figure 2 shows the $\phi$ yield divided by $N_{p p}$, the number of projectile participants, as a function of $N_{p p}$. The $\phi / N_{p p}$ ratio increases by a factor of 2.5 from peripheral to central collisions, which demonstrates that $\phi$ mesons are produced through collective phenomena, and not just a simple superposition of individual nucleon-nucleon reactions. Displayed in Figure 3 is the $\phi / K^{+}$ratio as a function of $N_{p p}$, where it is evident that the ratio is independent

of centrality. 


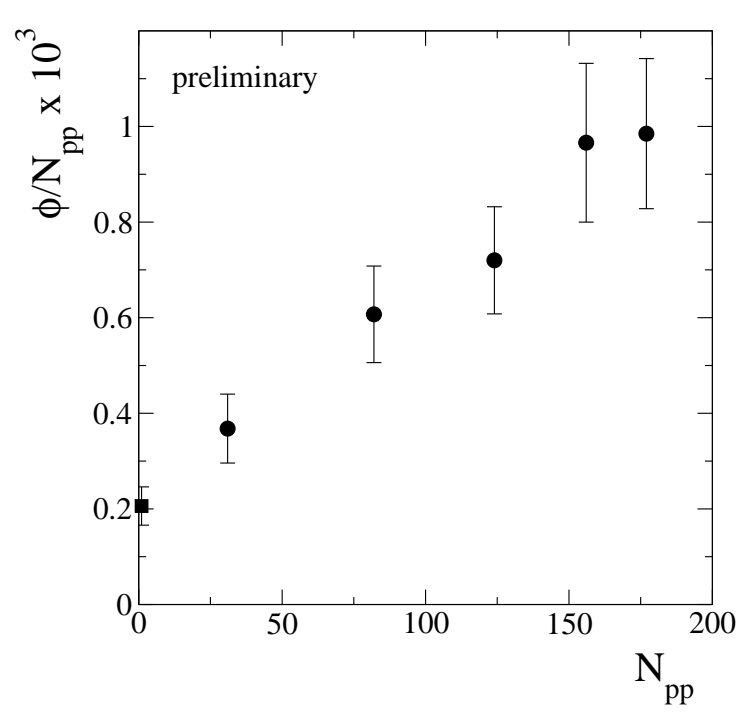

Figure 2. Ratio of $\phi$ yield to $N_{p p}$ as a function of $N_{p p}$. The circles are E917 data; the square is $\mathrm{p}+\mathrm{p}$ from Ref. [9].

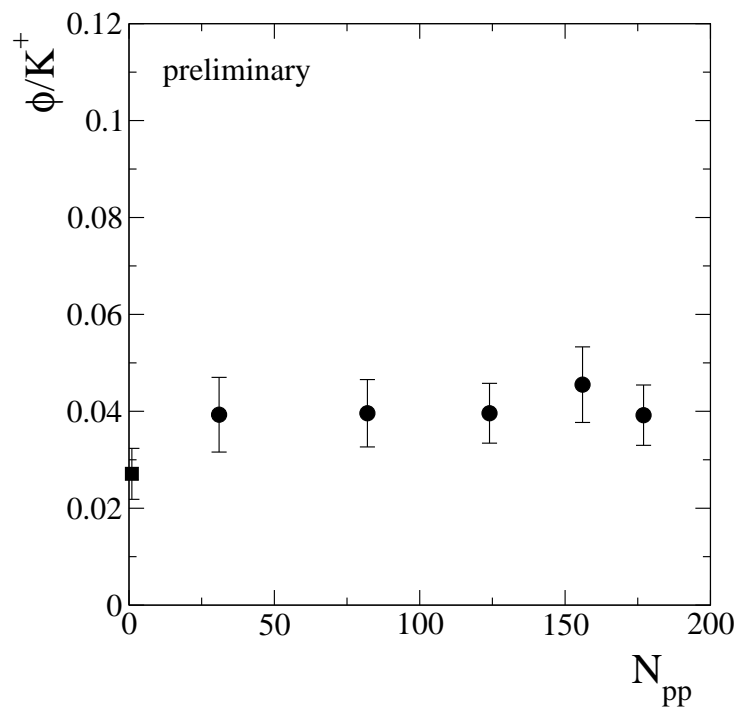

Figure 3. Ratio of $\phi$ to $K^{+}$yield as a function of $N_{p p}$. The circles are E917 data; the square is $\mathrm{p}+\mathrm{p}$ from Ref. [9].

\section{SUMMARY}

E917 has studied the systematics of baryon stopping, and the production of $\phi$ mesons, antilambdas, and antiprotons in $\mathrm{Au}+\mathrm{Au}$ collisions at 6, 8, and $10.8 \mathrm{GeV} /$ nucleon. These studies indicate that the colliding nuclei are not fully stopped, and that secondary interactions play a significant role in $\phi$ production. The large increase in the $\bar{\Lambda} / \bar{p}$ ratio from peripheral to central collisions remains unexplained by theory.

\section{REFERENCES}

1. L. Ahle et al., Phys. Rev. C57, 466 (1998).

2. T. Abbott et al., Nucl. Instr. and Meth. A290, 41 (1990).

3. B. Back et al., Phys. Rev. Lett. 86, 1970 (2001).

4. J. Cleymans, private communication.

5. F. Wang, private communication.

6. B. Back et al., nucl-ex/0101008.

7. T. Armstrong et al., Phys. Rev. C59, 2699 (1999).

8. W.-C. Chang et al., ICHEP2000 Proceedings.

9. W. Cassing and E.L. Bratkovskaya, Phys. Rep. 308, 65 (1999); A.A. Sibirtsev, Nucl. Phys. A604, 455 (1996). 